\title{
Mortalidad Materna
}

\section{(ESTUDIO DE 361 MUERTES CONSECUTIVAS)}

\section{Dr. Fernando Sánchez Torres*}

La mortalidad materna, al igual que la mortalidad perinatal, constituye un buen indicador de la calidad de los programas de protección materno--infantil. En países donde el nivel socio-cultural es alto y el cuidado a la mujer embarazada es de verdad asunto prioritario, pueden darse el lujo de preguntar si la mortalidad materna es hoy todavía un problema (4). En los países subdesarrollados o en vía de desarrollo, en cambio, la gestación suele pagar un gran tributo en vidas maternas, por diferentes causas, muchas de estas evitables, lo cual constituye ciertamente "un desafío para el obstetra y la comunidad", tal como lo plantean García y cols. (5).

En Colombia la magnitud exacta del problema no es posible establecerla por cuanto existe subregistro estadístico al no ser notificadas las muertes ocurridas a lo largo y ancho del país. Además, de los casios informados muy pocos están respaldados con el protocolo de autopsia y muchos tienen un certificado

\footnotetext{
* Profesor Titular del Departamento de Obstetricia y Ginecología. Facultad de Medicina (U. N.).
}

de defunción defectuosamente diligenciado. Por otra parte, no existe una entidad o dependencia oficial que se encargue del análisis detenido de las muertes maternas, con el fin de precisar sus causlas e identificar los distintos factores que puedan haber tenido participación en su ocurrencia.

Atendiendo las recomendaciones de la Federación Internacional de Ginecología y Obstetricia (FIGO), en 1971 comenzó a funcionar en el Instituto Materno Infanttil, de Bogotá, un Comité Permanente de Mortalidad. Materna constituído de la siguiente forma:

-Director Científico del Instituto. -Director del Departamento de Ginecología y Obstetricia.

- Jefe de la Sección Obstétrica.

-Patólogo.

-Representante de los Residentes.

EI objeto del presente trabajo es dar a conocer el rezultado del estudio de las muertes maternas sucedidas en el Instituto durante cinco años consecutivos (1971 1975). Conviene advertir que el Instituto Materno Infantil en un hos- 
pital universitario, con programas de pre y posgrado, adscrito al sector oficial. Además, es, en la capital del país, el único centro asistencial destinado a la atención obstétrica y ginecológica de la población carente de recursos económicos.

\section{Material y métodos.}

Ocurrida una defunción materna, el médico que asistió de último a la paciente estaba obligado a informar por escrito tal hecho a la Dirección del Instituto, en un formulario elaborado ad hoc, y solicitar la práctica de la necropsia.

Una vez que el protocolo de autopsia, junto con la respectiva historia clínica, llegaba a manos del Director, era convocado el Comité para considerar el caso. Si en el transcurso del análisis se encontrakan algunas dudas sobre el manejo médico, se citaban las personas que habían participado en él, con el fin de aclarar las dudas que pudiera tener el Comité. Adquirido por este procedimiento un concepto integral del caso, se procedía a calificar la evitabilidad o ineviitabilidad de la muerte y a señalar los factores que habían participado en su ocurrencia.

En relación con las definiciones y clasificaciones, nos sujetamos a las recomendadas por la FIGO (9), las cuales consideramos prudente transcribir:

\section{1. - Definiciones}

a) Muerte materna

"Es la muerte de una mujer, dekida a cualquier causa, mientras está embarazada o dentro de los 42 días que siguen a la terminación del embarazo, independientemente de la duración y del sitio del mismo".

b) Indice de mortalidad materna total.

"Es el número de muertes maternas (directas, indirectas y no obstétricas) por 10.000 nacidosi vi vos, considerando éste último término como el nacimiento de un producto vivo que pese 1.000 gramos o más. Las cifras que corresponden al numerador y al denominador deben corresponder al mismo período de tiempo".

c) Indice de mortalidad obstétrica.

"Se define como el anterior pero incluye solamente el número de muertes obstétricas directas e indirectas, es decir, excluyendo los casos de muerte obstétrica".

\section{2. - Clasificaciones}

a) Muerte obstétrica directa

"Es la debida a complicaciones obistétricas del estado grávico puerperal (embarazo, parto y puerperio) por intervenciones, omisiones, tratamientos incorrectos a una serie de eventos resultantes de cualesquiera de los anteriores".

b) Muerte obstétrica indirecta

"Es la resultante de una enfermedad preexistente que se desarrolla durante el embarazo y no se debe a causas obstétricas directas, pero que fue agravada por los efectos físiológicos del embarazo". 
c) Muerte no obstétrica.

"Es la que resulta de causas accidentales no relacionadas con el embarazo y su manejo".

Durante el lapso que abarca el presente estudio se registran 372 defiunciones maternas. Respetando lo establecido por la FIGO, hubimos de descartar once casos por haber ocurrido el deceso después de los 42 días de terminado el embarazo. La relación de estos casos es la sliguiente:

1. Murió por carcinoma metastásico, seis meses después de un aborto molar.

2. Murío 61 días después de una cesárea. Peritonitis.

3. Murió 52 días después de una laparatomía por embarazo ectópico.

4. Murió 66 días después por peritonitis posakorto.

5. Septicemia poscesárea. Murió 71 días después de la intervención.

6. Ruptura uterina. Peritonitis. Murió 56 días más tarde.
7. Aborto provocado. Murió 46 días después.

8. Apendicectomía durante el embarazo. Peritonitis. Falleció 75 días más tarde.

9. Aborto provocado. Septicemia Murió 48 días después.

10. Murió 70 días después del parto, por sepsiss.

11. Murió 49 días después de un aborto provocado. Septicemia.

En 272 de $\operatorname{los}^{\prime} 361$ casos analizados, se practicó autopsia, es decir, en el $75,35 \%$.

\section{Resultados.}

Durante los cinco años que abarca nuestro estudio ocurrieron 118.479 nacimijentos de niños vivos y se tuvieron en cuenta 361 muertes maternas. La tasa o índice de mortalidad materna fue, por lo tanto, de 30.47 por 10.000 nacidos vivos. En el cuadro $N^{\circ} 1$ puede observarse cuál fue el índice en cada uno de los años estudiados.

Cuadro No 1 - Tasas de mortalidad materna 1971-1975

\begin{tabular}{c|c|c|c|c|c}
\hline AÑO & 1.971 & 1.972 & 1.973 & 1.974 & 1.975 \\
\cline { 1 - 3 } TASA & 26.92 & 24.93 & 38.93 & 28.67 & 34.11 \\
\hline
\end{tabular}

Ochenta y dos $(22.7 \%)$ de las pacientes fallecidas eran primigestantes y $112(31 \%)$ eran grandes multíparas, es decir, habían tenido más de cinco partos No hubo defunciones en mujeres menores de 16 años; en cambio, 85 pacientes $(23.5 \%)$ eran mayores de 35 años.

La muerte ocurrió aún embarazada la paciente en 51 casos $(14.5 \%)$, en $136(37.67 \%)$ ya había 
sucedido el parto, y en 174 $(48.19 \%)$ se había producido un aborto.
Muertes obstétricas directas.

Se consideran como tales 315 defuncilones $(87.26 \%)$ por las causas señaladas en el cuadro $\mathrm{N}^{\circ} 2$.

Cuadro No 2 - Causas de muerte obstétrica directa

\begin{tabular}{|l|c|c|}
\hline \multicolumn{1}{|c|}{ C A U S A } & NUMERO & PORCENTAIL \\
Aborto & 163 & $45.15 \%$ \\
Toxemia & 64 & $17.73 \%$ \\
Sepsis posparto & 57 & $15.79 \%$ \\
Hemorragia obstétrica & 18 & $4.99 \%$ \\
Tromboembolismo & 9 & $2.49 \%$ \\
Anestesia & 3 & $0.83 \%$ \\
Accidente quirúrgico & 1 & $0.28 \%$ \\
\hline
\end{tabular}

Muertes obstétricas indirectas.

Con los inconvenientes, que anotaremos más adelante, encontrados para incluir en este grupo algunos casos, consideramos que $19(5.26 \%)$ podían tener cabida en este rubro. Las causas están consilgnadas en el cuadro No 3.

Cuadro $\mathbb{N}^{0} 3$ - Causas de muerte obstétrisc indirecta

\begin{tabular}{|l|c|c|}
\hline \multicolumn{1}{|c|}{ C A U S } & NUMERO & PORCENTAJE \\
Cáncer & 5 & $1.38 \%$ \\
Cardiopatias & 4 & $1.11 \%$ \\
Nefropatias & 3 & $0.83 \%$ \\
Aneurismas & 3 & $0.83 \%$ \\
Feocromocitoma & 1 & $0.28 \%$ \\
Anemia drepanocítica & 1 & $0.28 \%$ \\
Ruptura vena ovárica & 1 & $0.28 \%$ \\
Púrpura tromboci- & 1 & $0.28 \%$ \\
topénica & & \\
\hline
\end{tabular}


Muerte de causa no obstétrica.

En el cuadro No 4 se señalan las causas de la muerte en 27 casos
(7.48\%), que al criterio del Comité no estuvieron relacionadas con el estado grávido puerperal.

Cuadro No 4 - Muertes no obstétricas

\begin{tabular}{|l|c|c|}
\hline \multicolumn{1}{|c|}{ CA U S A } & NUMERO & PORCENTAJE \\
Neumonia & 7 & $\mathbf{1 . 9 4} \%$ \\
Apendicitis & 4 & $\mathbf{1 . 1 1} \%$ \\
Meningitis & 4 & $\mathbf{1 . 1 1} \%$ \\
Amibiasis & 3 & $0.83 \%$ \\
Suicidio & 3 & $0.83 \%$ \\
Traumatismos & 2 & $0.55 \%$ \\
Paludismo & 1 & $0.28 \%$ \\
Gangrena & 1 & $\mathbf{0 . 2 8} \%$ \\
\hline
\end{tabular}

Cuadro No 5 - Muertes evitables

\begin{tabular}{|l|r|c|}
\hline \multicolumn{1}{|c|}{ FACTOR } & NUMERO & PORCENTAIE \\
Paciente & 204 & $67.10 \%$ \\
Médico & 55 & $18.09 \%$ \\
Médico + Paciente & 19 & $6.25 \%$ \\
Médico + Institución & 15 & $4.93 \%$ \\
Institución & 6 & $1.97 \%$ \\
Médico + Pac. + Inst. & 5 & $1.61 \%$ \\
\hline
\end{tabular}

Establecidas las causas obstétricas directas e indirectas y descontadas las causas no obstétricas. puede decirse que el "índice de mortalidad obstétrica" fue de 28.19 por 10.000 nacidos vivos.

Evitabilidad.

Se consideró que 304 muertes
(84.21\%) eran evitables. El factor más implicado 204 ocasiones $(67.10 \%)$, fue el de la paciente misma, mientras el factor médico lo estuvo en 55 casos (18.09\%), el institucional en $6(1.97 \%)$ y el resto, $39(12.82 \%)$, fueron factores mixtos (cuadro $\mathrm{N}^{\circ} 5$ ). 


\section{Comentarios.}

Los resultados del presente estudio pueden considerarse altamente confiables por ser producto del examen detenido de cada caso de muerte materna y por estar respaldados con el $75.35 \%$ de autopsias.

En un estudio sobre mortalidad materna en el Instituto Materno Infantil durante los años 1954, 55, 56 y parte de 1957, Peralta y cols. (18) comunicaron haber contabilizado 174 defunciones sobre un to- tal de 28.428 nacidos vivos, es decir. que la tasa de mortalidad en aquel lapso fue de 60.12 por 10.000 nacidos vivos. Si la comparamos con la obtenida por nosotros, 30.47 , en el período de 1971 a 1975, inclusive, debemos aceptar que en el transcurso de 10 años la tasa de mortalidad materna se ha reducido a la mitad. Sin embargo, ha de aceptarse también que comparativamente con la tasia registrada en centros de maternidad de otros países, la del Instituto Materno Infantil, de Bogotá, sigue siendo alarmantemente alta (cuadro $\mathrm{N}^{\mathrm{o}} 6$ ),

\begin{tabular}{|c|c|c|c|c|}
\hline Autor & País & Institución & Años & Tasa mortalidad \\
\hline $\begin{array}{l}\text { Guha - Ray } \\
\text { (6) }\end{array}$ & U.S. A. & $\begin{array}{l}\text { Magee Women's Hos- } \\
\text { pital. }\end{array}$ & $1954-1974$ & 3. 44 \\
\hline $\begin{array}{l}\text { Krupip y cols. } \\
\text { (12) }\end{array}$ & U.S. A. & Charify Hospital & $1949-1969$ & 7. 55 \\
\hline $\begin{array}{l}\text { Agüero y cols. } \\
\text { (2) }\end{array}$ & Venezuela & $\begin{array}{l}\text { Maiernidad Concepción } \\
\text { Palacios. }\end{array}$ & $1964-1972$ & 12. 5 \\
\hline $\begin{array}{l}\text { García y cols. } \\
\text { (5) }\end{array}$ & Argentina & $\begin{array}{l}\text { Maternidad Provincial } \\
\text { de Córdoba }\end{array}$ & $1962-1969$ & 13.6 \\
\hline $\begin{array}{l}\text { Lozada y cols. } \\
\text { (13) }\end{array}$ & Perú & $\begin{array}{l}\text { Hospital General de } \\
\text { Arequipa. }\end{array}$ & $1971-1974$ & 17. 9 \\
\hline $\begin{array}{l}\text { Karchmer y } \\
\text { cols. (10) }\end{array}$ & México & $\begin{array}{l}\text { Hospital de Gineco } \\
\text { Obstefricia No. } 1\end{array}$ & $1961-1967$ & 24. 55 \\
\hline Sánchez & Colombia & $\begin{array}{l}\text { Instituto Maierno In- } \\
\text { fantil. }\end{array}$ & $1971-1975$ & 30.47 \\
\hline
\end{tabular}

Cuadro No 6 - Tasa comparativa de mortalidad materna con algunas instituciones extranjeras 
Es necesario comentar aquí que en todos los casos' se siguió la definición de muerte materna recomentada por la FIGO, la cual establece como límite los 42 días siguientes a la terminación del embarazo. Algunos aún utilizan la aprobada por el Comité sobre Atención Materno-Infantil de la Asociación Médica Americana que fija como límite 90 días (3). Si nosotros hubiéramos seguido esta definición, la tasa de mortalidad materna fuera de 31.40 por 10.000 nacidos vivos.

Si observamos el Cuadro No 1 podemos advertir que a lo largo de los cinco años estudiados la tasa de mortalidad materna no ha ido, como era de esperarse, en descenso sostenido; al contrario, mientras en 1971 fue de 26,92, en 1975 fue de 34.11. Más adelante podremos explicar este fenómeno.

El fallecimiento de las pacientes fue mucho más frecuente en la etapa posaborto $(48.19 \%)$ que en la de puerperio $(37.67 \%)$ y en la anteparto (14.5\%). Esta circunstancia se debe a las graves complicaciones que apareja el aborto provocado.

Dijimos antes que las muertes de causa "obstétrica directa" corresponden al $87.26 \%$ del total. Las causas más frecuentes fueron el aborto $(45.15 \%)$, la toxemia gravídica $(17.73 \%)$, la sepsis posparto $(15.79 \%)$ y la hemorragia obstétrica $(4.99 \%)$. Llama la atención, indudablemente, la elevada implicación del aborto como causa de muerte materna en el Instituto. Hace 20 años la muerte por sépsis, incluyendo la ocasionada por el aborto, ocupaba el tercer lugar en el grupo de muertes obstétricas directas, con un $16.66 \%$, mientras la toxemia encabezaba la lista (24.59$\%)$, seguida de cerca por la hemorragia obstétrica $(22.98 \%)$.

Si nos detenemos un poco más en el análisis de las 163 defunciones que ocasionó el aborto durante los cinco años estudilados, veremos que año por año aumenta: mientras en 1971 fue causa de 15 fallecimientos, en 1975 ocasionó 45 (Gráfica No 1). Solamente en tres casos el Comité tuvo la absoluta certeza $d_{e}$ que el aborto no habia sido deliberadamente inducido. En los restantes 160 hubo sospecha o evidencia de que había sido provocado. Son dignos de mención también los hechos de que el $12 \%$ fueran mujeres menores de 19 años y el $27 \%$ mayores de 35 . Asimismo, el $13 \%$ eran primigestantes y el $31 \%$ grandes multíparas (más de cinco partos). En 27 ocasiones el deceso ocurrió antes de completar 24 horas de hospitalizadas las pacientes, lo cual pone de presente la gravedad de su estado al ingreso.

En cuanto a la toxemia gravídica, a pesar de que ocupa el segundo lugar como causa de muerte materna, ha venido disminuyendo sostenidamente (Gráfica Nọ 2): Si en 1971 ocasionó 20 defunciones $(31.75 \%)$, en 1975 apenas murieron 8 mujeres por su causa $(9.88 \%)$. Un $78.13 \%$ de las muertes por toxemia se presentaron en pacientes con eclampsia, y el resto en preeclámpticas. 
Gráfica No. 1。- Mortalidad materna por aborto (1971-1975)

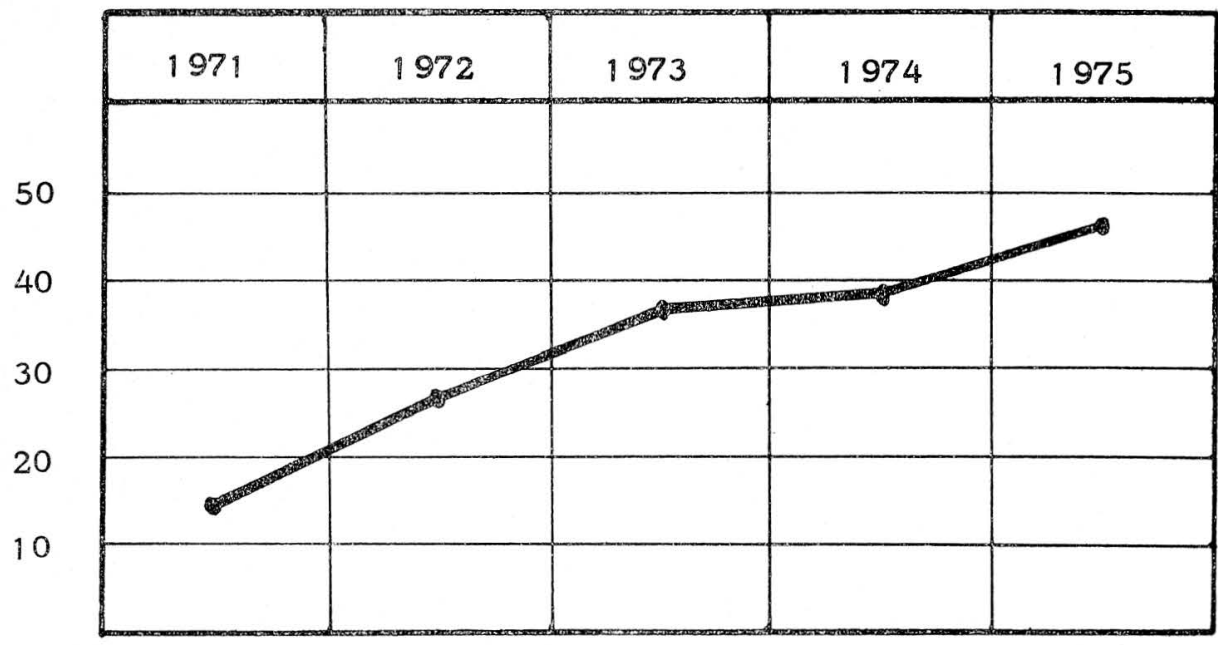

Gráfica No. 2. - Mortalidad materna por toxemia (1971-1975)

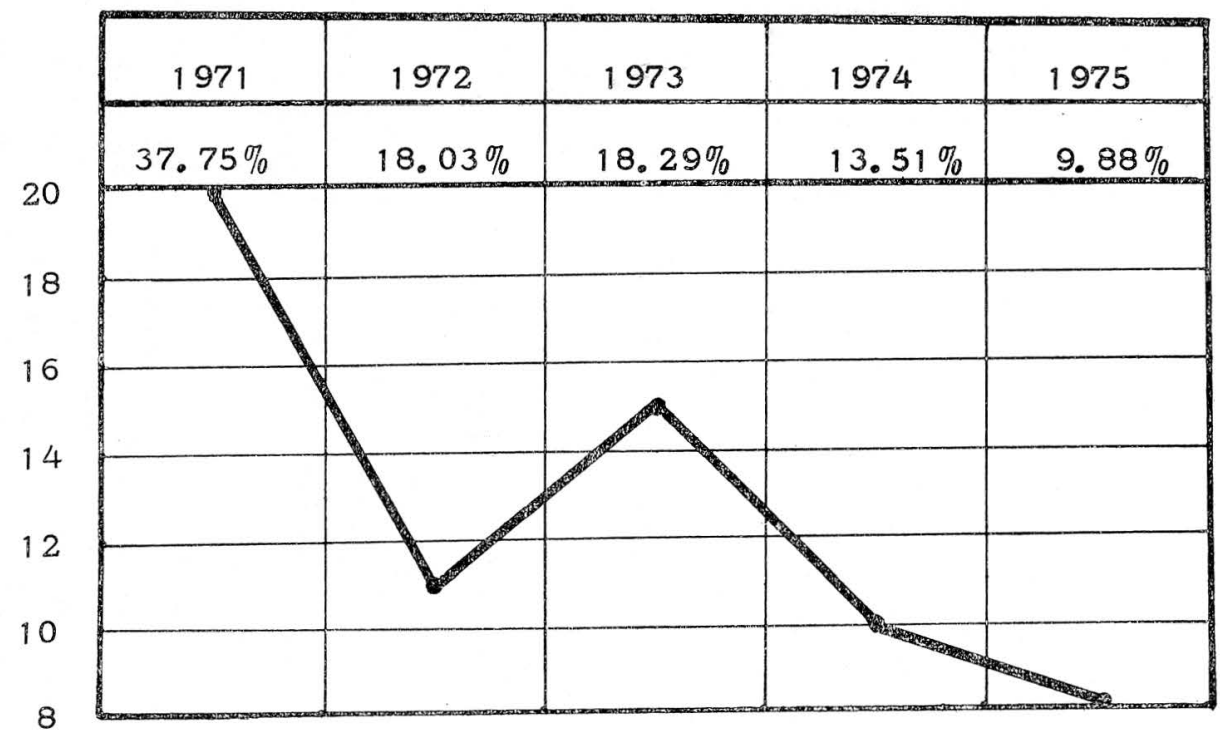


Sorprende el elevado número de muertes por sepsis puerperal $(15.79 \%)$. Aunque algunas mujeres ingresaron infectadas al hos. pital, hubo de aceptarse que la mayoría adquirió la infección durante el período de hospitalización. Indudablemente, el olvido de las normas de asepsia y antisepsia durante el curso del parto o de la operación cesárea, favorece la aparición de esta complicación. Tampoco hay que olvidar que el aumento de la infección es más frecuente en aquellas maternidades de carác: ter docente como el Instituto Materno Infantil.

La hemorragia de causa obstétrica, que 20 años atrás ocu jaba el segundo lugar $(22.98 \%)$ cayó al cuarto lugar $(4.99 \%)$. Esto permite afirmar que tanto el manejo obstétrico como los recursos para combatir la complicación h emorrágica se ha mejorado en el Instituto. La atonía y la ruptura uterina en grandes multíparas futron las principales causas de hemorragia.

Debido a la falta de claridad v precisión de la definición de "muerte obstétrica indirecta" recomendada por la FIGO, se hace difícil en muchos casos ubicar al.gunas muertes. Tienen razón Agüe ro y cols. (1) al afirmar que "la catalogación de las causas de muerte materna es uno de los insalvables escollos con que se tropiezan estos análisis, aun cuando se cuente con un alto porcentaje de autopzias". Igualmente, estamos de acuerdo con Karchmer y cols (10) cuando sugieren que la FIGO estructure unas definiciones más precisas que las vigentes.
Creyendo interpretar lo recomendado por la FIGO, solamente 19 casos fueron considerados como "muertes obstétricas indirectas" $(5.26 \%)$. En su orden, las causas más frecuentes fueron el cáncer, las cardiopatías y las nefropatías.

Respecto a las "muertes no obstétricas" se contabilizaron 27 casos $(7.48 \%)$ cuyas causas, en orden de frecuencia, fueron las siguientes: neumonía, apendicitis, meningitis, amibiasis y suicidios.

Aceptamos que juzgar acertadamente la evitabilidad o inevitabilidad de una muerte materna es a veces demasiado difícil, a pesar de que se disponga del mayor número de elementos de juicio. Quizás en ccasiones se actúe arbitrariamente. Sin embargo, para tratar de sacar algún provecho práctico al estudio de la mortalidad materna se hace necesario y conveniente establecer un juicio de responsabilidades. Es crobable que en uno o varios casos Ia responsabilidad de la muerte haya sido equivocadamente asignada, pero al hacer el balance en conjunto surgen factores de común ocurrencia, a los cuales se debe poner atención si se quiere combatir efectivamente la mortalidad materna.

En el presente estudio se consideró que el $84.21 \%$ de las muertes eran evitables (cuadro No 5), es decir, que de las 361 defunciones registradas solamente 57 han debido ocurrir, en circunstancias ideales. Si esto hubiera sucedido, la tasa de mortalidad materna que exhibiríamos sería de 4.81 y no de 30.47 . 
El factor más comprometido fue el relacionado con la paciente (204. casos). Seguramente se cometa una injusticia al echar la culpa de su muerte a la misma mujer, cuando ella es solo un producto del medio que la rodea. Las desfavorables condiciones sociales, culturales y económicas son en últimas las causas de esas defunciones. De ahí que tal vez fuera más razonable usar El término "ambiental extrahospitalario" para designar las fallas jnvolucradas en el factor que en nuestro estudio ha sido rotulado "paciente".

De las 304 muertes evitables, en $113(53.61 \%)$ la causa fue el aborto provocado. A este respecto hay que comentar que la disminución de las muertes por aborto no se ha logrado en el Instituto Materno Infantil a pesar de haber creado un Servicio dedicado exclusivamente al manejo de las complicaciones de esta entidad. Al contrario, como ya vimos, la mortalidad por aborto ha ido en aumento, es decir, que las condiciones ambientales extrahospitalarias desfavorables se han acrecentado.

Respecto al factor médico, en 55 casos $(18.09 \%)$ la muerte se atrikuyó a él. Errores de juicio y de técnica fueron los elementos que más contribuyeron a las muertes iatrogénicas. No olvidemos, como lo anotamos ya al comentar la sepsis intrahospitalaria, que el Instituto Materno Infantil es un centro asistencial universitario, y que por esta razón, aunque parezca paradójico, la morbilidad y mortalidad maternas se elevan.
El solo factor institucional se vio zomprometido en seis casos $(1.97 \%)$ y en 20 más $(6.57 \%$ ) asociado a los factores médico y paciente. La falta del equipo o del elemento necesario en el momento oportuno, como también los egresos demasiado precoces sin control domiciliario, fueron las fallas más frecuentes que se anotaron a dicho factor.

\section{Conclusiones y recomendaciones.}

De todo lo anterior podemos concluir que la Mortalidad Materna en el Instituto Materno Infantil es demasiado alta, y debe ser motivo de preocupación no solo para las directivas del hospital sino, particularmente, para los organismos oficiales de salud.

Si se tiene en cuenta que el factor paciente, relacionado íntimamente con la situación ambiental extrahospitalaria, contribuye al $67 \%$ de las defunciones, debe aceptarse que se requiere un cambio o mejoramiento de las condiciones sociales, culturales y económicas del núcleo de población que hace uso de los servicios del Instituto $\mathrm{Ma}$ terno Infantil. Asimismo, el incremento del aborto provocado hace n€cesario emprender campañas destinadas a reducir la frecuencia del em.tarazo indeseado mediante programas de educación sexual, como también mediante la prestación de servicios de contracepción, eficientes y de fácil acceso.

Nos identificamos con quienes afirman que si el riesgo materno materno puede ser establecido antes de la concepción, lo contracep- 
ción temporal o permanente contribuirá a la reducción de las muertes maternas $(7,14)$. Además es un imperativo reconocer los embarazos que se constituyen en riesgc para la mujer y concentrar en ellos toda la atención y cuidados (19). Por ejemplo, la gran multiparidad, al igual que la edad obstétrica avanzada de la mujer, son circunstancias que contribuyen a elevar notablemente las tasas de mortalidad materna $(8,11,15,16$, $17,20)$.

Por último, es de recomendar la creación de Comités de Mortalidad Materna a nivel institucional, pues con ellos además de ejercerse una auditoría médica, se establece un mecanismo para identificar las causas de las muertes maternas y los factores que intervienen en éstas.

\section{Resumen}

Se presentan los resultados del estudio exhaustivo de las muertes maternas ocurridas en el Instituto Materno Infantil, de Bogotá, duran. te un período de cinco años consecutivos 1971 a 1975, inclusive) En este lapso se registraron 372 fallecimientos, pero se descartaron once for haber ocurrido después de 42 días de terminado el embarazo. El estudio estuvo a cargo de un Comité Permanente de Mortalidad Materna y los diagnósticos finales estuvieron respaldados en el $75 \%$ de los casos por el respectivo pro. tocolo de autopsia.

La tasa de mortalidad materna encontrada fue de 30.47 por 10.000 nacidos vivos. De acuerdo a la cla- sificación de la FIGO, 315 muertes $(87.26 \%)$ fueron consideradas de causa "obstétrica directa", siendo las más frecuentes el aborto provocado $(45 \%)$, la toxemia gravídica $(17.7 \%)$, la sepsis posparto $(15.8 \%)$ y las hemorragias obstétricas (5\%). De causa "obstétrica indirecta" se clasificaron 19 casos $(5.26 \%)$, señalándose como las más frecuentes las cardiopatías, el cáncer y las nefropatías. Se registraron, además, 27 casos de muerte "no obstétrica" (7.5\%), acupando los primeros lu gares la apendicitis, la neumonía y la meningitis.

El $84 \%$ de las muertes fueron catalogadas como "evitables", atribuyéndose su ocurrencia al factor paciente en el $67 \%$ de los casos, aj factor médico en el $18 \%$ y el fac. tor institucional en el $2 \%$. El resto se debió a factores mixtos.

Se pone de presente, como conclusiones, que la tasa de mortalidad encontrada es demasilado alta, tratándose de un hospital universitario. Sin embargo, al estudiar los factores implicados en las muertes se deduce que en éstas influyen más los aspectos social, cultural y económico, aue el simplemente institucional. El aborto provocado, que ha tenido un auge alarmante, se encuentra involucrado en casi la mitad de las defunciones, en tanto que la toxemia gravídica y las he morragias obstétricas han ido disminuyendo.

Por último, se recomienda la creación de comités permanentes de mortalidad materna a nivel instituclional, pues al tiempo que se constituyen en un mecanismo de auditoría médica, son un valioso instru- 
mento para identificar las causa: de muerte y los factores que constituyen a ésta. En esta forma se hace más fácil emprender medidas encaminadas a disminuir las defunciones ocurridas en la etapa grávido-puerperal.

\section{SUMMARY}

The results of an exhaustive study about the maternal deaths accurred in the Instituto Materno Infantil de Bogotá, during a period of five consecutive years (1971 to 1975 inclusive) are presented in this arti. cle, During this time 372 passing: registered but eleven were rejectec because they occured after 42-nd days of their pregnancy being over

The study was carried out by $\varepsilon$ Commission on Maternal Mortality which was permanent and its final diagnosis in $75 \%$ of the cases was backed up by the respective recorded autopsias.

The index of maternal mortality they found was 30.47 for 10.000 newborns alive. According the clas. sification of FIGO, 315 death cases $(87.26 \%)$ were considered of "direct obstetric cause" being provoked abortion the most frequent $(45 \%)$, the gravilidic toxemia $(17,7 \%)$, the post labor sepsis $(15.8 \%)$, and obstetric bleeding
$(5 \%)$, Ninteen cases were classified as of "indirect obstetric cause" (5. .26\%) being the most common cardilopaties, cancer and nephropàties. Besides 27 cases of on obstetric death were register $(7,1 \%)$, where appendicitis, neumoni: anmeningitis were of importan consideration. $84 \%$ of the cases was classified as "avoidable" where $67 \%$ was ascriked to the patient $18 \%$ to the medical personnel and $2 \%$ to the ilnstitution.

As a conclusion, it can stated that the mortality index found is very high since it is a university hospital. Nevertheless wen studyng the involved factors in the death cases in can be said that the soclial, cultural and economic aspects play a more important role than simply the institutijoanl aspect. The provoked abortion that every day is more common causes almost half of the deceases whille the gravidic toxemia and obstetric bleedings had decreased in quantity. Finally, it can be recommended the foundation of permanent commissions at an institutional level because while they become a medical audit mechanism, are an important tool to identify the causes and factors associated wilth maternal death. In this way it can be easier to undertake the necessary measures to deceases occured in the stage gravidic puerperal. 


\section{BIBLIOGRAFIA}

1. Agüero, O.; Torres, J. I. Aure, M. "Tendencias de la mortalidad mater na en la Maternidad Concepción Pa lacios (1939-1963)". Rev. Obst. Gin. Venezuela 26:505, 1966.

2. Agüero, O.; Torres, J. I. y Kizer, S. "Mortalidad materna en la Maternidad Concepción Palacios (1964-1972) Ginec. Obstet. Méx. 35:509, 1974.

3. American Medical Association. "A Guide for Maternal Death Studies Committee on Maternal and Child Care of the Council on Medical Service" 1957.

4. Dietel, H. y Keding, G. "Müttersterblichkeit-auch heute noch ein Problem?" Geburtsh. U: Frauenheilk. 36:1, 1976.

5. García, H. H.; Ortiz, S. y Blank, B. "Mortalidad materna. Un desafío para el obstetra y la comunidad", Obst. y Gin. Lat. Am. 29:478, 1971.

6. Guha-Ray, D. K. "Maternal mortality in an urban hospital. A fifteen year survery". Obst \& Ginec. 47:430, 1970.

7. Gibbs, C. E. y Locke, W. E. "Maternal deaths in Texas, 1969 to 1973".Am. J. Obstet. \& Gynecol. 126: 687, 1976.

8. Hellman, L. M. y Pritchard, J. A. "Abnormalities of the puerperium". En Wiliams Obstetrics, 14 th edition. New York Appleton- Century Crofts, pag. 971 - 1006. 1971.

9. International Federation of Ginecology and Obstetrics. "Report on the activity of the Committee on Maternal Mortality". J. Int. Fed. Gynaec. Obstet, 5:302, 1967.

10. Karchmer, S.; Armas-Domínguez, J. Chávez-Azuela, J. y Shor, V. "Estu. dios de mortalidad materna en México. Consideraciones médico sociales". Gaceta Med. de México. 109:63, 1975.
11. Klein, M. D. y Karten, I. "Maternal deaths: A heath and socioeconomic challenge". Am. J. Obsst. \& Gynec. 110, 298, 1971.

12. Krupp, P. J.; Barclay, D. L. Roleing. W. M. y Wegener, G. "Maternal mortality. A 20 year study of Tulane Departament of Obstetrics an Gynecology at Charity Hospital". Obst \& Gynec. 35:823, 1970.

13. Lozada, B. y Salinas, L. "Mortalidad materna en el Hospital General de Arequipa". Rev. Peruana de Gin. y Obst. 15:69, 1969.

14. Marmol, J. G.; Vollman, R. F.; Gordon, M., Patterson, S. P. y Reid, W. A. Maternal death and high-risk pregnanc an analysis of 40 maternal deaths in the collaborative proyect". Obst. \& Gynec. 30:816, 1967.

15. Nortman, D. "Parental age as a factor in pregnancy outcome and child development". Reports on Population Family Planning. N․ 16, pag. 52, 1974.

16. O'Sullivan, J. F. "The hazards associated with multipary" J. Obst. \& Gynec. Brit. Comm. 70: 158, 1963.

17. Oxforn, H. "Hazards of grand multipary". Obst. \& Gynec. 5:150, 1955.

18. Peralta, R.; Silva, C. R., Pérez, E. y Ramírez, J. "Mortalidad materna". Rev. Colombiana de Obst. y Gin. 9:1. 1958.

19. Phillips, O. C.; Hulka, J. F. Vincent, M. y Christy, W. C. "Obstetric mortality: A 26-year survey". Obst. \& Ginec. 25:217, 1965.

20. Radovic, P. "Frequent and high parity as a medical and social problem". Am. J. Obst. \& Gynec. 94:583, 1966. 